\title{
RÉFLEXIONS
}

\section{SUR LA PROTECTION DE LA FORÊT MÉDITERRANÉENNE CONTRE L'INCENDIE}

En 1963, nous avons assisté et même participé à la création de l'" Entente interdépartementale en vue de la protection de la forêt méditerranéenne contre l'incendie". Pour son $25^{\mathrm{e}}$ anniversaire, nous avons présenté à son Assemblée générale une note de réflexion sur les progrès apportés depuis à la protection de la forêt.

Les lecteurs de la Revue forestière française ne sont que très exceptionnellement des familiers de ces questions. C'est pourquoi nous leur présentons ici un résumé de cette note. Nos propos souvent élogieux mais, parfois aussi, critiques ne visent pas à convaincre mais plus modestement à provoquer la réflexion du lecteur.

Tout d'abord, il taut insister sur l'importance qu'a eue la création de l'Entente. Nous ignorons pourquoi le Groupement aérien a été récemment soustrait de son autorité pour dépendre directement du ministère de l'Intérieur (sans doute un ancien réflexe de centralisation). Mais il faut rappeler que ce fut l'Entente elle-même qui créa le groupement.

En outre l'existence de l'Entente a eu une grande importance politique. Elle a donné son unité à un problème qui se posait séparément à quinze départements. Elle a affirmé leur volonté d'en finir avec le fléau des feux de forêts.

\section{***}

Nos réflexions seront présentées en quatre thèmes.

\section{LE RÔLE DE L'OPINION PUBLIQUE}

Pour des raisons économiques connues, la protection de la forêt méditerranéenne est essentiellement l'affaire des collectivités publiques. Cela la rend étroitement dépendante de l'opinion publique que motive la grande presse et qui nous vaut des crédits de plus en plus importants pour assurer cette protection. Récemment, c'est dans les mêmes conditions qu'a été présenté un projet en vue de reboiser le massif de la Sainte-Victoire ravagé l'an dernier par le feu.

Mais par contre, le débroussaillement autour des habitations, mesure d'un grand intérêt technique, est impopulaire auprès des propriétaires. Alors nous déplorons que les responsables, fonctionnaires ou élus, aient négligé trop souvent de le faire exécuter et cela depuis vingt-trois ans! 
II faut donc porter une grande attention aux informations propagées par les média. II faut surtout réfléchir au moyen de les rectifier. Elles sont parfois dangereuses.

- Ainsi il est inexact de dire que la forêt est dégradée par des incendies de plus en plus étendus et qu'elle est condamnée à disparaître. Au contraire, il faut faire savoir qu'elle est de mieux en mieux protégée et notamment que les incendies de 1989, qui ont été jugés particulièrement graves par le public, l'ont été nettement moins que la moyenne brûlée annuellement il y a vingt-cinq ans.

- Il faut dire que la malveillance n'est pas la seule cause importante des incendies comme le public le croit trop souvent. Les imprudences ont une très lourde responsabilité.

- Il faut contester que l'incendie serait un mal récent créé par le développement du tourisme. Les champions de cette opinion (dont certains investis parfois de lourdes responsabilités) devraient consulter à cet égard le Centre de Documentation du Tholonet.

- Il faut rappeler que la forêt est cantonnée par l'agriculture sur des terres ingrates et qu'il est bien souvent utopique de vouloir la faire cloisonner par des cultures. L'élevage par contre peut être parfois organisé d'une façon efficace pour détruire la broussaille. Mais sa rentabilité pose alors encore bien des problèmes.

- Les moyens aériens sont certes très efficaces. Mais nous ne saurions sous-estimer leurs points faibles: impossibilité d'intervenir de nuit, danger du vent... Surtout l'éloge que nous en faisons ne doit pas nous faire oublier le rôle essentiel des pompiers.

- Il est inexact de parler du débroussaillement comme d'une panacée sans préciser qu'il ne peut concerner que des surfaces limitées et doit être associé à d'autres équipements du terrain : vigies, chemins, points d'eau...

- Nous faussons les idées quand nous confondons, sous le terme de "prévention ", les mesures destinées à "éviter l'éclosion du feu " avec les équipements du terrain destinés à "faciliter la lutte ". En effet, c'est une règle applicable à la protection contre tous les accidents, quelle que soit leur nature : le bon sens veut que priorité soit donnée à la "prévention". Or, la confusion que nous dénonçons ici laisse croire que la stricte prévention des incendies est dotée de plus de crédits qu'elle ne l'est en réalité, même si à cet égard la situation s'est améliorée au cours des dernières années.

- Mais l'erreur essentielle est la confiance excessive que nous avons en notre pouvoir d'éteindre les grands feux. Personne n'est responsable des échecs qui sont alors constatés, personne ne doit avoir honte de cette situation. II n'existe pas d'outil adapté à cette tâche. Tout au plus pouvons-nous éteindre un grand feu quand il vascille ou en empêcher la reprise après qu'il se soit éteint. Cela n'est déjà pas si mal. Cessons donc de rêver à des victoires coûteuses, dangereuses et illusoires contre les grands feux.

Toutes les erreurs que nous venons de signaler sont imputables à une information incomplète du public. Elles ont pesé et pèsent parfois encore sur les décisions des responsables surtout lorsqu'une administration centralisée les tient trop éloignés des réalités du terrain.

\section{LA COMPLEXITÉ DE L’ORGANISATION DEMANDE UNE SÉRIEUSE COORDINATION}

II y a vingt-cinq ans, en forêt méditerranéenne comme dans toute la forêt française, la protection contre l'incendie était l'affaire des propriétaires, du maire, des pompiers, des forestiers et des gendarmes.

Tout cela s'est bien compliqué.

Un partenaire nouveau est intervenu: le Service météorologique.

De plus, ont été créés:

- une Entente interdépartementale,

- des chantiers d'anciens harkis,

- des groupements de sapeurs forestiers, 
- des comités communaux feux de forêts,

- un service statistique spécialisé,

- une école de la Sécurité civile,

- un centre de documentation sur la forêt méditerranéenne,

- un centre interrégional de coordination des secours,

- un détachement spécial de la Sécurité civile,

- un détachement aérien d'engins bombardiers d'eau,

- un comité scientifique et technique des feux de forêts,

- une association et une revue Forêt méditerranéenne,

- enfin, de nombreuses associations ou syndicats de propriétaires forestiers.

Ces organismes sont originaux et particuliers à la forêt méditerranéenne. Ils sont généralement indépendants. Leurs responsables sont souvent jaloux de leurs prérogatives. Chacun a un rôle utile. Mais pour le jouer, il doit tenir compte du rôle du voisin. Pour cela, il devrait bien le connaître. Ce n'est pas toujours le cas.

Pour répondre à cette préoccupation, il faut une coordination. Pour permettre en outre des interventions rapides, il faut que cette coordination soit traduite par un plan, un plan élaboré dès la base, la commune ou le secteur de commune, précis, détaillé...

Certes cela n'a pas été totalement méconnu:

- Les réunions du Conseil de l'Entente sont ouvertes et sans pour autant voter, de nombreux responsables y assistent auprès des Conseillers généraux.

- Les pouvoirs du Préfet de Marseille, déjà très étendus en ce qui concerne la Sécurité civile, ont été confirmés et élargis pour la lutte contre les incendies.

- Enfin, nous pouvons constater qu'il y a une bonne volonté de coordination aux échelons locaux. Mais la difficulté subsiste pour la coordination entre des directions ministérielles très centralisées et parfois entre les corps de fonctionnaires.

C'était en vue d'améliorer encore cette situation qu'avaient été créés : une Mission interministérielle de Coordination, ainsi qu'un programme finalisé dans le Plan d'action économique et sociale.

Ces organismes d'État ont joué un rôle appréciable. Cependant ils ont été supprimés en 1982 , et cela bien que les directions ministérielles des Forêts et de la Sécurité civile soient demeurées centralisées.

Ils ont été remplacés par:

- le Conservatoire de la Forêt méditerranéenne,

- et le Conseil consultatif d'Orientation de la Forêt méditerranéenne.

Ce sont là deux organismes qui dépendent eux aussi de l'État et sont centralisés. Toutefois, leur installation à Marseille auprès du Préfet de Région paraît marquer une volonté de les rapprocher des réalités locales. Cependant, à en juger d'après leur comportement, ils ne sont que très peu ouverts au public. Cela convient mal à la situation.

En effet, les pompiers et les personnels du groupement aérien ont une tâche dangereuse et pénible; les forestiers et les gendarmes ont également un travail difficile. En outre, sur un terrain accidenté et boisé, il n'est pas toujours possible à leurs supérieurs de suivre à vue leurs interventions qui, par ailleurs, doivent être très rapides. Enfin, le public, qui circule en forêt, a lui aussi des responsabilités. Compte tenu de cette situation, il faut que les uns et les autres soient sérieusement motivés. Cela ne sera possible que s'ils connaissent bien et comprennent l'organisation à laquelle ils sont associés. II faut donc les associer au mieux à la préparation de la protection. II est encore temps.

Dans ces conditions, les délibérations du Conservatoire et du Conseil consultatif devraient être ouvertes au public ou au moins faire l'objet de communiqués de presse. 


\title{
LA CHASSE AUX BAVURES
}

Les statistiques nous montrent que sur les trois ou quatre mille incendies qui dévastent tous les ans la forêt méditerranéenne, quelques dizaines à peine atteignent la centaine d'hectares. Ainsi les $9 / 10$ de la surface brûlée sont imputables à quelques rares grands feux.

Cette observation est à rapprocher de la remarque faite ci-dessus: nous sommes incapables d'éteindre les grands incendies. II peut donc suffire d'une légère erreur pour qu'un feu n'ait pas pu être maîtrisé. Ce sont quelques bavures qui nous valent les tristes bilans de la statistique. Elles peuvent être des erreurs d'équipement en matériel, d'aménagement du terrain, de transmission, d'engagement des moyens... Même avec une bonne organisation et un personnel de qualité, de telles erreurs sont toujours possibles. Il faut les débusquer pour corriger tous les ans le plan local de protection. L'enquête statistique réglementaire doit alors être complétée par une étude de responsabilité. II est peu probable que les grands feux soient de bons sujets d'étude. La lutte, nous l'avons vu, est trop confuse. Elle suscite trop de controverses. Mieux vaudrait alors compter sur les feux d'une centaine d'hectares. II faudrait simplement savoir pourquoi ils n'ont pas été arrêtés à quelques dizaines d'hectares comme c'est le cas normalement.

Cette étude sera délicate. Les fautes probables concerneront tout le dispositif et tous les organismes responsables. Mais les pompiers qui sont en bout de chaîne de ces responsabilités risqueront d'être les boucs émissaires. Aussi c'est au Préfet, parfois peut-être au Gouvernement, de composer la commission d'enquête et d'en confier la présidence à une personnalité impartiale, souvent même à un pompier.

Les résultats de ces enquêtes serviront tous les ans à corriger les plans locaux de protection à l'échelon de la commune ou du secteur de commune.

\section{LA RECHERCHE EN VUE D'AMÉLIORER LA PROTECTION}

Aujourd'hui malgré les améliorations obtenues, les résultats ne sont pas encore satisfaisants. II est donc normal que chaque organisme cherche à améliorer ses méthodes de travail et mène pour cela des études de sa propre initiative et avec ses moyens. Mais, lorsque cette recherche demande des moyens exceptionnels ou lorsqu'elle concerne plusieurs organismes, il faut la normaliser. Pour cela, l'organisme payeur doit exiger un protocole d'accord élaboré par les demandeurs avec l'accord du Comité scientifique et technique des feux de forêts. Cela écartera les initiatives irréfléchies, souvent inutilement onéreuses.

Enfin le suivi des résultats demande souvent plusieurs années. II doit alors être en charge de la statistique Prométhée. Pour cela, dans le secteur géographique soumis à une expérience, les formulaires de l'enquête statistique de routine seront complétés.

C'est ainsi que peu à peu les idées jugées les plus séduisantes pour l'amélioration du dispositif pourront être testées sérieusement. À cet égard, bien que les secteurs de débroussaillement ne soient plus du strict domaine expérimental, il serait bon d'en suivre encore l'efficacité de la même façon.

Les réflexions que nous venons de formuler ne constituent pas un projet de réorganisation de la protection. Plus modestement, elles sont une simple série de remarques à l'intention des responsables. Car l'ensemble de l'organisation est encore trop récent pour pouvoir être jugé comme parfaitement rodé.

\author{
A. SEIGUE \\ Ingénieur général du GREF (e.r.) \\ 6, La Pelissière \\ 12, boulevard de Gabes \\ 13008 MARSEILLE
}

